\title{
Sherrie Elzey and De-Hao Tsai win ABC Best Paper Award
}

\author{
Nicola Oberbeckmann-Winter
}

Published online: 14 May 2014

(C) Springer-Verlag Berlin Heidelberg 2014

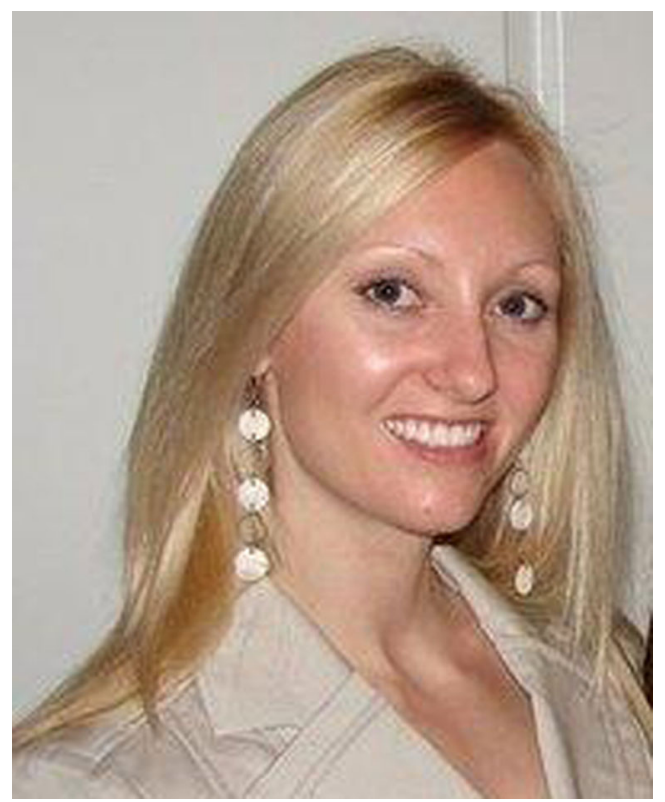

Sherrie Elzey was born in 1981. She earned her B.S. degree in chemistry and B.A. degree in physics from the University of Northern Iowa (Cedar Falls, IA, USA). She received a National Defense Science and Engineering Graduate fellowship and earned her $\mathrm{Ph} . \mathrm{D}$. degree from the University of Iowa (Iowa City, IA, USA). She was then awarded a National Research Council postdoctoral fellowship at the National Institute of Standards and Technology (NIST; Gaithersburg, MD, USA). At NIST, she conducted research in the Materials Measurement Laboratory focusing on method development for measuring the size and elemental composition of representative therapeutic nanoparticles with functionalized surfaces. After her postdoctoral research, she joined TSI (Shoreview, MN, USA) as an applications engineer, where she applied a variety of sizing methods to measure airborne and liquid-phase nanoparticles. She is currently a regional sales manager at TSI.

N. Oberbeckmann-Winter $(\bowtie)$

Analytical and Bioanalytical Chemistry, Springer-Verlag GmbH,

Tiergartenstraße 17, 69121 Heidelberg, Germany

e-mail: abc@springer.com

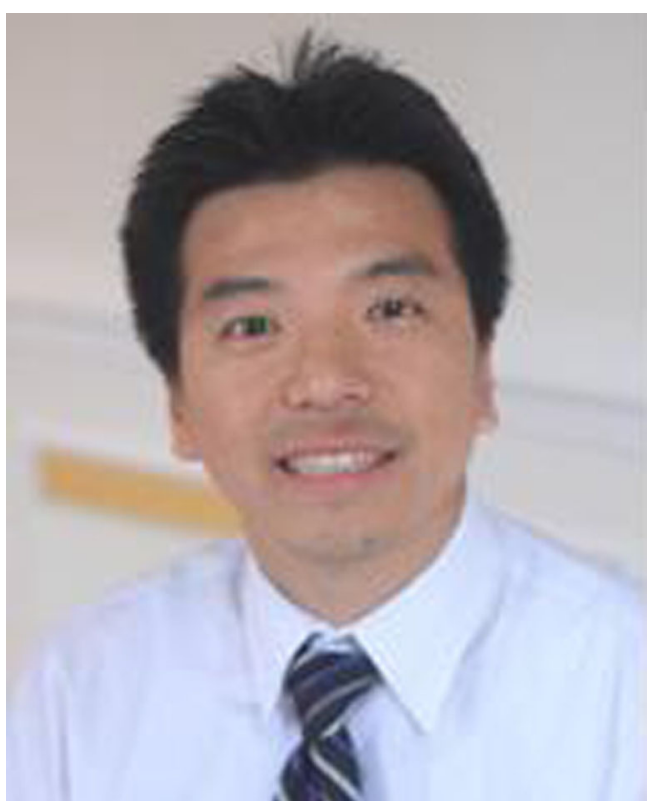

De-Hao Tsai was born in 1976. He earned a B.S. degree in chemical engineering from the National Cheng Kung University (Tainan, Taiwan) in 1998, and an M.S. degree in chemical engineering from the National Tsing Hua University (Hsinchu, Taiwan) in 2000. He received his Ph.D. degree in materials science and engineering from the University of Maryland (College Park, MD, USA) in 2007 under the supervision of Michael R. Zachariah. He is currently Assistant Professor in the Department of Chemical Engineering at NTHU. Prior to joining NTHU, he worked as a research scientist at Cabot Microelectronics (Aurora, IL, USA; 2008-2009) and as a research associate at NIST (2009-2013). During his tenure at NIST, his research work focused on measurements for applications in nanomedicine supervised by Vincent A. Hackley. In 2013, he received the 2013 Distinguished Associate Award for Technical Achievement at NIST to acknowledge his exemplary leadership in the development of innovative physical and chemical metrology for applications in cancer nanotechnology.

The ABC Best Paper Award 2013 for outstanding work published in Analytical and Bioanalytical Chemistry (ABC) goes to Sherrie Elzey (32 years old) and De-Hao Tsai (37 years old), who are lead authors of the article "Real-time size 
discrimination and elemental analysis of gold nanoparticles using ES-DMA coupled to ICP-MS," which presents the development of a method to characterize simultaneously the size and elemental composition of nanoparticles, especially those used for nanotherapeutics. Sherrie Elzey and De-Hao Tsai demonstrate a proof of concept for a measurement method that determines the elemental composition of size-separated particles, thereby providing both size and chemical information from a single coupled system. This analytical approach is broadly applicable to both chemical and biochemical research, as well as product development, for example, consumer products, biomedical and diagnostic devices, and nanocoatings.

Accompanied by $€ 1,500$, the award is sponsored by Springer to honor exceptional young scientists and to stimulate their research careers. The article "Real-time size discrimination and elemental analysis of gold nanoparticles using ESDMA coupled to ICP-MS" by Sherrie Elzey, De-Hao Tsai, Lee L. Yu, Michael R. Winchester, Michael E. Kelley, and Vincent A. Hackley was published as a Paper in Forefront and is freely available online during 2014 at http:// link.springer.com/article/10.1007/s00216-012-6617-z or can be requested as a PDF file.

\section{Who are Sherrie Elzey and De-Hao Tsai?}

Sherrie Elzey and De-Hao Tsai talk about their research, their motivations, and themselves to Nicola OberbeckmannWinter.

\section{What was your motivation to do research in the area of nanoparticle analysis?}

Sherrie: When I was considering my research options in graduate school, nanoscience was a new concept to me. It sounded like an interesting interdisciplinary field that fit well with my background in chemistry and physics.

De-Hao: On graduating with a Ph.D. degree, I realized how important it was to have suitable metrology and methods to correlate the material properties of nanoparticles with their performance. Hence, over the past few years, my research has been devoted to the area of nanoparticle analysis.

\section{Why do you think your work merits the $\mathrm{ABC}$ Best Paper} Award?

Our work demonstrates a method that can provide numberand mass-based distributions, elemental composition, particle concentration, and nanoparticle density. It can be applied widely for many different applications.
How does the award-winning work relate to your Ph.D. research and your present work?

Sherrie: I used both the size separation method and elemental analysis methods to characterize nanoparticles during my graduate research. In my present work, I interact with researchers using these and other analyses to study particles for a broad range of applications.

De-Hao: I have been working on stand-alone particle sizing [electrospray differential mobility analysis (ES-DMA)] since my Ph.D. studies. The work presented in our article gave me the opportunity to learn how to analyze simultaneously the number concentration and elemental composition of sizeresolved nanoparticles through the coupling of ES-DMA with inductively coupled plasma mass spectrometry. I think the work is also very helpful to my present work on quantifying the loading of metallic drug ligands on nanoparticle surfaces and also the composition of nanoparticle assemblies.

\section{How would you explain your research to your child?}

We use high voltages and high temperatures to measure particles that are too small to see. This helps people understand what the particles are and how they can be used.

\section{What is the trickiest problem you have had to overcome in your research? How did you solve it?}

Because this work coupled different techniques, we had to be sure that the calculations based on data we extracted from the measurements were valid. In other words, we had to be careful that our assumptions were valid and we were considering all sources of error in the measurements. In this respect, it was critical to take advantage of the interdisciplinary nature of the work. Several colleagues from different divisions provided reviews and helpful discussions throughout this research.

Where do you see your field headed and how do you see it influencing bioanalytical research?

The field of nanoparticle metrics is trending toward real-time analysis based on methods that can provide complementary data. As these metrics are developed, nanoparticle properties will be better understood and therefore better utilized for specific applications. One area in particular that will benefit from such methods is nano-based therapeutics. 
Which incident/discovery has proved most valuable for your own research? Which incident/discovery most inspired you during your education and scientific career? Which recent discovery might prove most valuable to the field of (bio)analytical research or beyond?

Sherrie: The development of differential mobility analysis for size separation of nanoparticles has influenced and inspired my career. I used this technique for research during my graduate and postdoctoral work, and I use it in my current career.

De-Hao: The first time I saw nanoparticles aligning on a charge-patterned substrate under a scanning electron microscope was very inspiring. At the time, I believed I could use electrostatic force to direct and classify nanoparticles, even though I could not see them visually. For bioanalytical research, I think the discovery of using ES-DMA to characterize ligand-conjugated gold nanoparticles was very valuable and provided an effective way to obtain a full particle size distribution and the coating thickness of the molecular corona on particles.

\section{What was the best/worst advice you ever received?}

Sherrie: A phrase that I recall often is "So what? It's no big deal." It helps me to refocus when I start to worry about whether I am making a mistake or if I might fail. Most mistakes are really no big deal; they are just learning experiences. When I am making a decision, I envision the worst-case scenario and then ask myself "so what?" This helps me make sure I am not avoiding anything for fear of failure.

De-Hao: The best advice for me is "try more; don't give up". In many cases I can get through problems because of it.
Whom do you admire the most or who inspired you the most and why?

Sherrie: My graduate advisor, Vicki Grassian. It was inspiring to work with and learn from a successful, driven woman and see how she worked hard and collaborated with other researchers. During my time at the University of Iowa, she established the Nanoscience and Nanotechnology Institute. It was great to see how a project such as that is started and developed.

De-Hao: My Ph.D. advisor, Michael Zachariah, and my NIST supervisor, Vincent Hackley. They had a positive impact on my research work. I learned a lot about nanoscience and nanotechnology from, and was inspired by, Michael Zachariah when I was his graduate student at the University of Maryland. Vincent Hackley was a role model for how to conduct a research project in a systematic way, and showed me how to be missiondriven to ensure our team could achieve its goal-the work presented in our award-winning article is a great example.

\section{What are your future plans?}

Sherrie: I really enjoy learning, so I am always open to continuing my education and seeing what opportunities are available. I am open to trying different career paths.

De-Hao: My career plan is to be a good teacher at my university and also a great scientist in the field of nanoparticle research.

\section{What do you do in your spare time?}

Sherrie: I like to get outside when the weather is nice-jogging, hiking, playing with my dog, grilling. The other half of the year in Minnesota, I sit by the fireplace and watch movies.

De-Hao: I like to travel for leisure and/or for conferences, whenever I have spare time. 